\title{
Spinal Deformity Correction in Duchenne Muscular Dystrophy (DMD): Comparing the Outcome of Two Instrumentation Techniques
}

\author{
Ujjwal Kanti Debnath, Syed M. Hossein Mehdian, John K. Webb \\ The Centre for Spinal Studies and Surgery, Queens Medical Centre, Nottingham NG7 2UH, UK
}

\begin{abstract}
Study Design: A retrospective matched cohort study.
Purpose: To compare the results of combined Luque rod-sublaminar wiring (thoracic) and pedicle (lower lumbar) instrumen tation (SLW) versus those with pedicle screw fixation (PS) for scoliosis correction in Duchenne muscular dystrophy (DMD). Overview of Literature: PS fixation is gaining popularity. Two instrumentation systems were not compared before in a matched cohort of patients.

Methods: Two groups of patients with DMD were matched according to the age at surgery, magnitude of deformity and vital capacity. Indications for surgery included loss of sitting balance, rapid decline of vital capacity and curve progression. In group 1 ( 22 patients) SLW fixation was used from T2/3 to pelvis or sacrum. In group 2 (18 patients) PS fixation was used from T2/3 to L5. Five patients had all level segmental PS fixations. Minimum follow-up was 2 years (range, 2 to 13 years). Radiographs, lung function tests and subjective/objective assessment were performed at standardized intervals.

Results: Mean Cobb angle in group 1 improved from $45.3^{\circ}$ (range, 26 to $75^{\circ}$ ) to $17.7^{\circ}$ (range, 0 to $37^{\circ}$ ) and mean pelvic obliquity improved from $14.5^{\circ}$ (range, 8 to $28^{\circ}$ ) to $5.6^{\circ}$ (range, 0 to $15^{\circ}$ ). Mean Cobb angle in group 2 improved from $42.8^{\circ}$ (range, 28 to $80^{\circ}$ ) to $7.3^{\circ}$ (range, 0 to $20^{\circ}$ ) and mean pelvic obliquity improved from $11.2^{\circ}$ (range, 7 to $30^{\circ}$ ) to $2.0^{\circ}$ (range, 0 to $\left.5^{\circ}\right)(p<0.05)$. Mean operating time and blood loss were less in group 2 ( $p<0.05$ ). In group 1 , the infection rate and instrumentation failure was higher, and subjective/objective outcomes showed no significant difference between the groups.

Conclusions: PS fixation had superior correction and controlled pelvic obliquity without the need for pelvic fixation.
\end{abstract}

Key Words: Muscular dystrophy, Duchenne, Spinal deformity, Scoliosis, Pedicle Instrumentation, Sub laminar wiring

\section{Introduction}

Progressive scoliosis develops in most patients with Duchenne muscular dystrophy (DMD) due to paralysis of the extensor muscles [1]. The progression can be rapid leading to serious deterioration of unsupported sitting ability and deterioration of the respiratory function already compromised by the disease $[2,3]$. Spinal stabilization is required to prevent these disabilities. Spinal instrumentation in DMD has seen a sea of change over the last five decades [4-14].

The trend in the 1980s was to extend the fixation to the pelvis or sacrum. However, the prolonged operating time and increased blood loss persuaded many to stop the distal instrumentation at L5 level [10,15-17]. Most current literature deals with the instrumentation of the spine using hooks and wires or hybrid constructs with pedicle screws in lum-

Received Sep 9, 2010; 1st Revised Nov 23, 2010; 2nd Revised Dec 3, 2010; Accepted Dec 6, 2010

Corresponding author: Ujjwal Kanti Debnath, FRCS (T\&O), DM (Orth)

The Centre for Spinal Studies and Surgery, Queens Medical Centre, Nottingham NG7 2UH, UK

23, Barons Court Road, Cardiff CF23 9DF, UK

Tel: +44-7811165794, Fax: +44-2920456355, E-mail: ukdeb86@ hotmail.com 
bar region. Although, the use of pedicle screw constructs in thoracic spine for idiopathic scoliosis was established in late 90s [18-20], it was not until recently, Hahn et al. [21] reported for the first time the outcome of patients following use of only pedicle instrumentation in DMD.

The present study compares the clinical and radiographic results of combined Luque rod-sublaminar wiring (thoracic) and pedicle screws (lower lumbar) (SLW) versus only pedicle screw fixation (PS) in two consecutive patient cohorts with scoliosis in DMD.

\section{Materials and Methods}

The data for two-matched consecutive cohorts were collected retrospectively at a single institution (University Hospital, Nottingham). These patients were treated within a span of 17 years from 1990-2007. Group 1 consisted of the historic cohort of 22 patients treated between 1990 and 2000 with Luque rod-sublaminar wiring with pelvic fixation with either Galveston or L-rod technique (SLW group). Group 2 consisted of the cohort of 18 patients who were treated between 2000 and 2007 with pedicle screw con- structs (PS group). The senior authors (SHM and JKW) were involved in treating patients from both groups.

The groups were matched according to three criteria: similar age at surgery, similar magnitude of spinal curvatures (all type 1 and 2) [22], and vital capacity. Indications for surgery were loss of sitting balance, presence of scoliosis with or without evidence of curve progression and diminishing vital capacity. Both groups had similar operative exposures and upper thoracic fixation levels.

In group 1, the correction was achieved using Luque rods fixed with sublaminar wires in the thoracic and lumbar spine (Fig. 1). The Galveston technique was used in 6 cases and the L-rod configuration in 16 cases for pelvic fixation.

\section{Technique}

Pedicle screws are first placed in the lumbar spine. Posterior superior iliac spine is identified and the outer wall of ilium is dissected in a subperiosteal plane to reach the greater sciatic notch. This position acts as a reference for insertion of the rod on either side. The rods are contoured and prebent to realign the deformed spine. The basic princi-
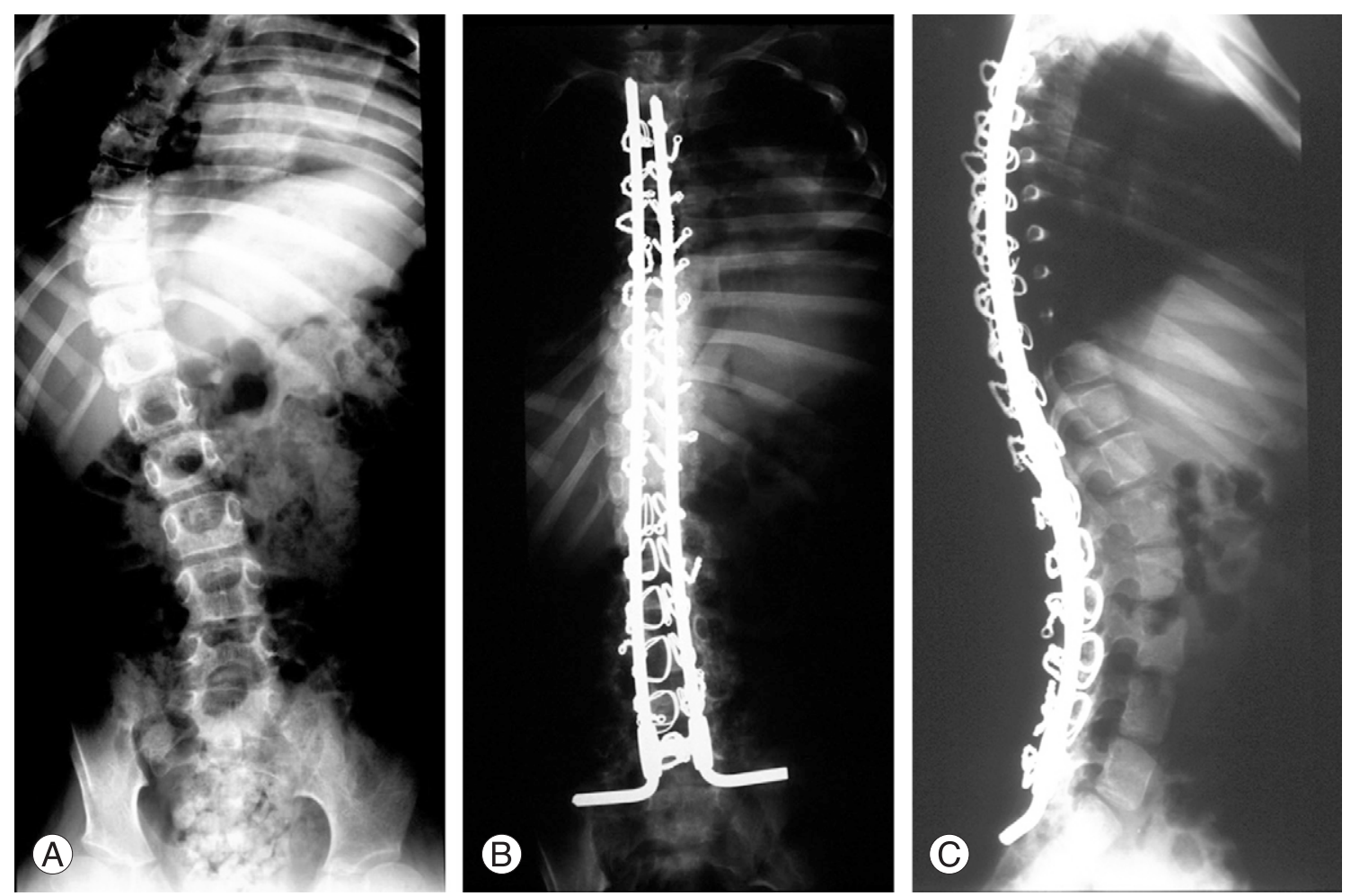

Fig. 1. A 11-year-old boy, wheelchair bound for 8 months with Duchenne muscular dystrophy. (A) Preoperative antero-posterior radiograph right sided showing $60^{\circ}$ curve. (B, C) Two year post-operative antero-posterior and lateral radiographs showing sublaminar wiring instrumentation with Luque rods and distal fixation to pelvis with L-rod configuration. 
ple determining the sequence depended on where the initial correcting load needed to be applied. Once the rods are fixed to the lumbar pedicle screws (as the foundation) a cantilever corrective load (that is, one that has support at one end) to the rod or rods was applied, their unattached ends are brought to the anchorage at the opposite end. A strong foundation is defined as strong enough to accept corrective loads and to resist deforming loads without dislodgment of the anchors or plastic deformation of the rods. The thoracic foundations consisted of instrumentation spanning at least three consecutive vertebrae. The instrumentation sequence was initiated on the convex side of the thoracolumbar curve and then added on the concave side, with the cephalad rod connected side to side to the intrailiac post (Galveston) anchorage or L rod configuration. Two short closed loop sublaminar wires were used to fix the rods to the spine.

In group 2 fixation of the spine was carried out with pedicle screws in the thoracic as well as in the lumbar spine (Fig. 2) and to L5 vertebrae in all the patients. Pedicle screws with a diameter of $5 \mathrm{~mm}$ and $6 \mathrm{~mm}$ were used in the thoracic and the lumbar spine respectively. Anatomical landmarks were used to insert the screws. In 5/18 patients all level segmental pedicle screw construct was used and the rest had alternate segmental pedicle screw constructs in which every other vertebra in the main lumbar region and every second or third vertebra in the upper thoracic region were instrumented. The average number of pedicle screws used was 24 (range, 18 to 34 ) per patient. Following instru- mentation all had autologous bone graft and supplemental femoral head allograft for fusion following standard facet excision and decortication. All patients had intra-operative neurophysiologic monitoring (SSEPs and SMEPs). Postoperatively, all the patients were managed in a pediatric intensive care unit until they no longer required any ventilatory support.

The following clinical data were recorded: forced vital capacity (FVC) and forced expiratory volume in the 1st second estimated immediately preoperatively and at final follow-up, electrocardiogram findings, echocardiogram results, weight, age at surgery, estimated blood loss, operation ("skin to skin") time, complications and the duration of hospitalization following surgery.

Antero-posterior and lateral radiographs of the whole spine in sitting were taken in all patients preoperatively and during the follow-up. The Cobb angle of the scoliosis and the pelvic obliquity were measured from the radiographs preoperatively, 6 months postoperatively and at the final follow-up. An independent assessor assed the radiographs and measurements were made independently of the surgeons involved. Functional assessment was performed using the EK scale [23]. The EK scale comprises of ten categories (EK1-10) which was developed to determine the need for and impact of intervention in the non-ambulatory stages of DMD. The questionnaire incorporates 10 items which deal with the patients ability to use wheelchair, ability to transfer from wheelchair, ability to stand, ability to balance in the wheelchair, ability to move the arms, ability
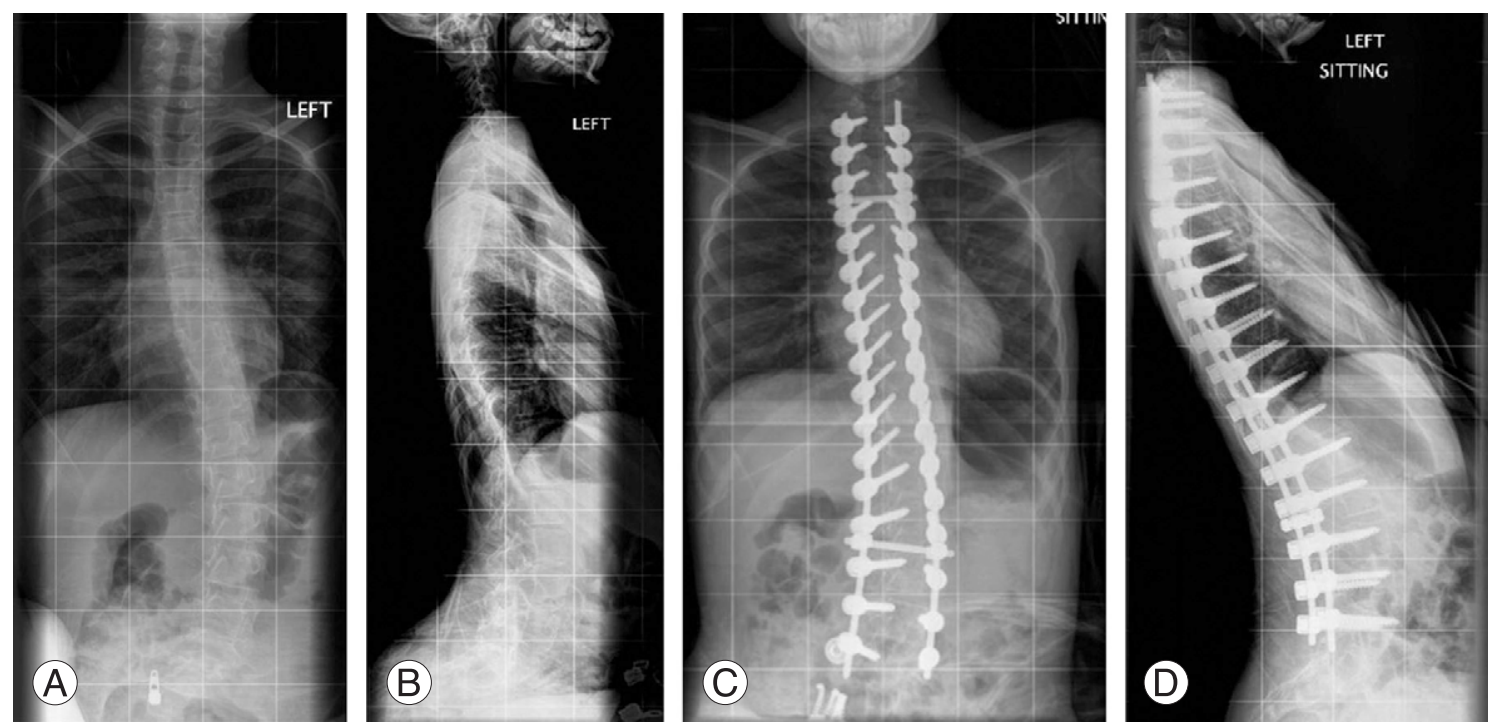

Fig. 2. A 13 year-old-boy wheelchair bound for 2 years with Duchenne muscular dystrophy. (A, B) Preoperative antero-posterior and lateral radiographs showing left sided scoliosis with $40^{\circ}$ curvature. (C, D) Two year post-operative antero-posterior and lateral radiographs showing instrumentation with pedicle screws. 
to use the hands and arms for eating, ability to turn in bed, ability to cough, ability to speak and physical well being. A range of scores can be possible from 0-30. Lower the score better the function.

\section{Statistical analysis}

Pre and post-operative and final follow-up radiographs were used to analyse the Cobb angle and pelvic tilt. Postoperative corrections were analysed using paired t-test. Similarly correction percentage at initial post-operative radiographs and final follow-up radiographs was compared using paired $t$-test $(p<0.05$ was considered to identify significant differences). We determined the duration of operation, mean blood loss and mean post-operative hospital stay to see if these factors were associated with better outcome with chi square test.

\section{Results}

\section{Group 1}

DMD was diagnosed at a mean age of 4.5 years (range,
1.5 to 9 years). The patients became wheelchair bound at a mean age of 9.6 years (range, 6 to 12.5 years) and operated at a mean age of 12.5 years (range, 9 to 16 years). At the time of surgery, the FVC was $57 \%$ (range, 34 to $72 \%$ ). The scoliosis was thoracic in 2 cases, thoracolumbar in 14 cases and lumbar in 6 cases. The mean body mass index (BMI) was $22.5 \mathrm{~kg} / \mathrm{m}^{2}$. The mean follow-up in this group was 4.5 years (range, 3 to 9 years). The mean Cobb angle was $45.3^{\circ}$ (range, 26 to $75^{\circ}$ ) and the mean pelvic obliquity was $14.5^{\circ}$ (range, 8 to $28^{\circ}$ ) at the time of surgery. Immediately after surgery, the mean Cobb angle measured $16^{\circ}$ (range, 0 to $35^{\circ}$ ) and at final follow-up was $17.7^{\circ}$ (range, 0 to $37^{\circ}$ ) (Fig. $3 \mathrm{~A})$. The immediate postoperative pelvic obliquity measured $5.7^{\circ}$ (range, 0 to $15^{\circ}$ ) and at final follow-up was $5.6^{\circ}$ (range, 0 to $15^{\circ}$ ). The mean operative time was 260 minutes (range, 180 to 380 minutes), mean estimated blood loss was 3.41 (range, 2 to 10.01 ), mean intensive care stay was 56 hours (range, 48 to 80 hours) and mean hospital stay was 8.5 days (range, 6 to 16 days). Two patients had excessive intra-operative bleeding treated with blood transfusion. Five patients had infection (four superficial and one deep). Three patients required prolonged ventilatory support but didn' $t$ require any tracheotomy. Two patients had implant failure
Distribution of Cobb angle-Group 1

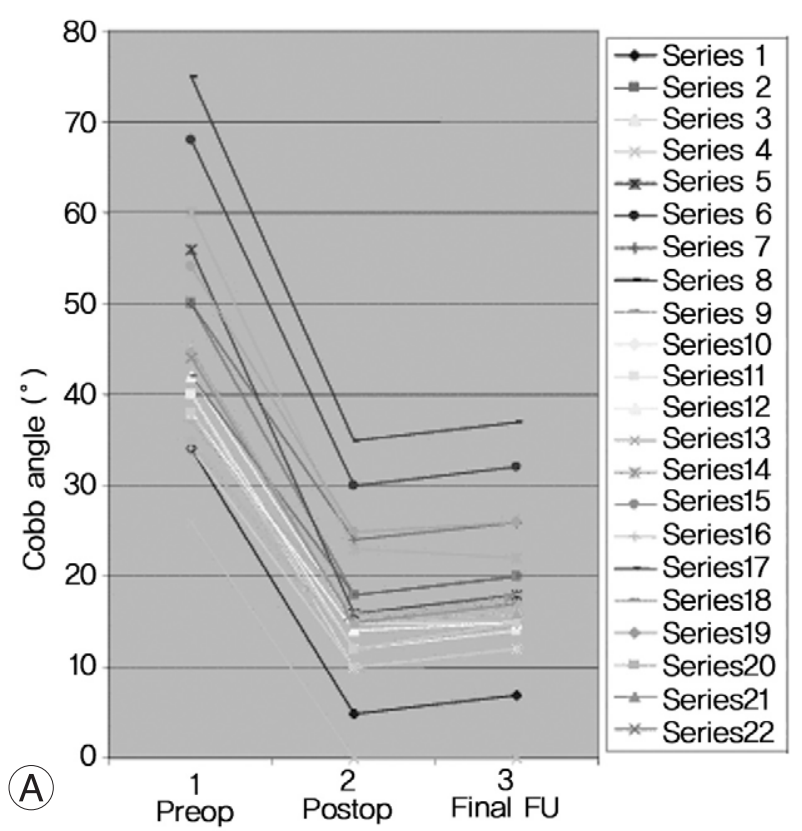

\section{Distribution of Cobb angle-Group 2}



Fig. 3. (A) The distribution of Cobb angle at the time of surgery, immediately after surgery, and at final follow-up in individual cases in group 1 (SLW). (B) The distribution of Cobb angle at the time of surgery, immediately after surgery, and at final follow-up in individual cases in group 2 (PS). SLW: Luque rod-sublaminar wiring (thoracic) and pedicle (lower lumbar) instrumentation, PS: Pedicle instrumentation, Preop: Pre-operative, Postop: Post-operative, FU: Follow-up. 
(one had loosening of the implant and the other had proximal junctional kyphosis). Pelvic obliquity was maintained at or below $10^{\circ}$ in all but two patients.

\section{Group 2}

The mean age of diagnosis in this group was 5 years (range, 4 to 12 years). The patients became wheelchair bound at a mean age of 9.8 years (range, 6 to 13 years) and operated at a mean age of 11.8 years (range, 9 to 15 years). At the time of surgery, the FVC was 53\% (range, 34 to $78 \%$ ). Fifteen patients had $\mathrm{C}$ shaped curve with equal distribution of sidedness. The curves were mainly thoracic in 3, thoracolumbar in 13 and lumbar in 2 patients. The mean follow up in this group was 2.3 years (range, 2 to 6 years). The mean BMI was $25.4 \mathrm{~kg} / \mathrm{m}^{2}$. The mean Cobb angle was $42.8^{\circ}$ (range, 28 to $80^{\circ}$ ) and the mean pelvic obliquity was $11.6^{\circ}$ (range, 0 to $20^{\circ}$ ) at the time of surgery. Immediately after surgery, the mean Cobb angle measured $4.5^{\circ}$ (range, 0 to $15^{\circ}$ ) and at the final follow-up was $7.3^{\circ}$ (range, 0 to $20^{\circ}$ ) (Fig. 3B). The immediate postoperative pelvic obliquity measured $2.6^{\circ}$ (range, 0 to $6^{\circ}$ ) and at final follow-up was $2^{\circ}$ (range, 0 to $5^{\circ}$ ). The mean operative time was 216 minutes (range, 180 to 300 minutes), mean estimated blood loss was 2.01 (range, 1 to $3.7 \mathrm{l}$ ), mean intensive care stay was 44 hours (range, 36 to 72 hours) and mean hospital stay was 7 days (range, 6 to 14 days). Two patients had infection treat- ed with drainage, irrigation and antibiotics. One patient had broken rod and loose screw, which was revised.

Comparing the two groups it was observed that although both groups had surgery for similar curve magnitudes, the mean curve magnitude at final follow up was significantly higher in group $1(p<0.01)$ (Table 1). The percentage of curve correction and pelvic obliquity correction was significantly higher in the group $2(p<0.01)$. Intra-operative instrumentation was more time consuming in group $1(p<$ $0.01)$. Intra-operative blood loss was significantly higher in group $1(p<0.01)$. The functional outcome as measured by EK scale was similar in both groups. The mean EK score was 8.95 (range, 2 to 23) in group 1 as compared to 8.72 (range, 3 to 19$)$ in group $2(p=0.8)$.

\section{Discussion}

The scoliosis correction in DMD is challenging because of the primary muscular pathology and the frequently associated reduced pulmonary reserve. Several instrumentation techniques has evolved over the last five decades since Harrington [6] first reported the use of rods and hooks in spinal fusion for DMD. This was discarded in early 70s due to multiple complications encountered in various series $[6,9,22]$. Luque [8] introduced sublaminar wiring in early 80 s for spinal stabilization in deformities which was undertaken in many centres with variable success. The authors

Table 1. A comparison of the key mean data from the two groups

\begin{tabular}{lccc}
\hline \hline Data & Group 1 (SLW) & Group 2 (PS) & $p$-value \\
\hline No. of patients & 22 & 18 & - \\
Mean age at diagnosis (yr) & $4.5(1.5-9)$ & $5(4-12)$ & - \\
Mean age at surgery (yr) & $12.5(9-16)$ & $11.8(9-15)$ & - \\
Mean preoperative FVC $(\%$ pred. value) & $57(34-72)$ & $53(34-78)$ & - \\
Mean preoperative Cobb angle (range, $\left.{ }^{\circ}\right)$ & $45.3(26-75)$ & $42.8(28-80)$ & - \\
Mean preoperative Pelvic tilt angle (range, $\left.{ }^{\circ}\right)$ & $14.5(8-28)$ & $11.2(0-20)$ & - \\
Flexibility Index (range) & $62.8(54-70)$ & $65.5(57-72)$ & - \\
Mean body mass index $\left(\mathrm{kg}^{\circ} / \mathrm{m}^{2}\right)$ & 22.5 & 25.4 & $<0.01^{\text {a) }}$ \\
Mean final Cobb angle $($ range, & $\left.{ }^{\circ}\right)$ & $7.3(0-20)$ & $<0.01^{\text {b) }}$ \\
Mean final pelvic tilt angle (range, $\left.{ }^{\circ}\right)$ & $17.7(0-37)$ & $2(0-5)$ & $<0.01^{\text {b) }}$ \\
Mean \% Cobb angle correction (range) & $5.6(0-15)$ & $83(77-89)$ & $<0.01^{\text {b) }}$ \\
Mean \% pelvic tilt correction (range) & $62(51-70)$ & $83(73-97)$ & $<0.01^{\text {b) }}$ \\
Mean operative time in min (range) & $63(45-81)$ & $216(180-300)$ & $<0.01^{\text {a) }}$ \\
Mean blood loss in 1 (range) & $260(180-380)$ & $2(1-3.7)$ & $<0.01^{\text {a) }}$ \\
Mean intensive care stay (hr) & $3.4(2-10)$ & $44(36-72)$ & $<0.05^{\text {a) }}$ \\
Mean hospital stay in days (range) & $56(48-80)$ & $7(6-14)$ & - \\
\hline
\end{tabular}

SLW: Luque rod-sublaminar wiring (thoracic) and pedicle (lower lumbar) instrumentation, PS: Pedicle screw fixation, FVC: Forced vital capacity.

${ }^{\text {a) }} \boldsymbol{x}^{2}$ test, ${ }^{\text {b) }}$ Paired $t$-test. 
reported a mean correction of deformity on the frontal plane equal to $50 \%$, and only the severest cases required postoperative immobilization. The advantages of surgery that they described include increased comfort and sitting tolerance [1,4,5,8,10-12]. More recently, Cervellati et al. [24] recommend the use of Luque rods and sublaminar wiring since it ensures a satisfactory, stable and durable correction of the deformity with good restoration of trunk balance.

In 1984, Allen and Ferguson [25] proposed a modified system of the segmental spinal instrumentation, known as the Galveston technique. This modification provided better fixation and correction of pelvic obliquity. However, they pointed out that pelvic fixation is a technically demanding procedure that increases operative time and risk. The fixation to pelvis was the norm for better sitting stability. Sussman [12] suggested that instrumentation and fusion to L5 might be adequate in DMD. Fusion to the fifth lumbar vertebra has been recommended [15] in cases in which pelvic obliquity (measured as the angle between a line tangential to the superior margins of both iliac crests and a line perpendicular to one through the spinous processes of L4 and L5), is less than $10^{\circ}$, and the Cobb angle is less than $40^{\circ}$. Sengupta et al. [17] recommended L5 fixation for young patients with smaller curves and minimal pelvic obliquity. Bony fusion to the pelvis is difficult to achieve because of the severe osteopenia in patients with DMD. Increased blood loss is another intra-operative complication which is dreaded by surgeons and anesthetist. In the current series i.e., SLW group had significantly increased operating time as well as blood loss which confirm the observation of others [26]. This was due to bleeding from epidural vessels during the subperiosteal dissection for passage of the sublaminar wire.

Since the introduction of segmental pedicle fixation in idiopathic scoliosis in mid 90s, many surgeons are using pedicle fixation to good use in DMD patients $[19,20,26]$. Hahn et al. [21] reported the first series of twenty patients treated with pedicle instrumentation from T3/T4 to the pelvis. Pelvic fixation was done with iliac screws similar to Galveston technique [14]. The mean total blood loss was 3.71 , intensive care unit stay was 77 hours and hospital stay was 19 days. The mean Cobb angle correction was $77 \%$ from $44^{\circ}$ to $10^{\circ}$, pelvic obliquity improved $65 \%$ from $14^{\circ}$ to $3^{\circ}$. In our series, lower mean blood loss of 21 was recorded, intensive care unit stay was maximum of 48 hours and mean hospital stay was 7 days in the PS group. The radiographic results in this series of pedicle instrumentation were comparable to the series presented by Hahn et al. [21]. This series had a mean Cobb correction of $83 \%$ and pelvic obliquity correction of $83 \%$. The pelvic obliquity correction achieved was much higher as compared to others. It is an interesting difference since the pedicle instrumentation group didn't have any pelvic fixation as described for every patient in the series presented by Hahn et al. [21]. It is presumed that the reason for such a difference may be due to the fact that their series had patients who had higher pelvic obliquity (at least five patients had more than $20^{\circ}$ pelvic obliquity).

Alman and Kim [15] questioned the use of pelvic fixation of rods since the pelvic obliquity progressed in $78 \%$ of their patients despite pelvic fixation. But they also recommended that if the apex of the curve is caudal to L1, one may use pelvic or sacral fixation. They further suggested the use of hooks or screws in the lumbar region to avoid the failures seen in sublaminar wiring. Use of pedicle screw system for DMD was started in this institute since the turn of the millennium with an idea to avoid the complications observed in the sublaminar wiring. It was observed in our practice that pedicle screw system had better intra-operative control of the correction of the curve in sagittal and coronal plane as compared to the sublaminar wiring. Gayet et al. [27] introduced the flexible spine instrumentation with rods fixed to the lumbar and sacral spine using screws. They achieved good correction with improved spinal growth by $5 \mathrm{~cm}$. Thus, the quest for better instrumentation techniques continued until the pedicle screws were found to be biomechanically superior since they are penetrating anchors as compared to the gripping fixation obtained by wires and cables $[28,29]$.

Kim et al. [30] compared two techniques i.e., pedicle instrumentation and hybrid system of sublaminar wiring and lumbar pedicle screws in idiopathic scoliosis. They found similar results in junctional changes, lowest instrumented vertebra, surgical time and clinical outcome. Although, similar curve magnitudes were observed in both surgical groups in this series, the curve correction (both in coronal and sagittal plane) achieved was much higher in the pedicle instrumentation group [26]. These results obtained in DMD patients points out that pedicle instrumentation has a better deformity correction force and probably maintains the correction even without pelvic or sacral fixation. Good functional outcome was evident in five patients who had high BMI and had pedicle instrumentation at all segments from T2 to L5. Increased body weight owing to steroid 
intake in these patients may be benefited by pedicle instrumentation at all levels since they may be at risk of implant failure. This precludes the need for revision surgery in these patients who has little longevity.

In this study, both the groups were matched for mean age at surgery, mean spinal curvature and diminishing vital capacity. The matching study cohorts make it easier to interpret the results. In one series, three methods of instrumentation techniques were compared but none of the groups were matched. The number of subjects in each group was less and authors had to interpret the results of curve correction with caution [26].

The risks involved in pedicle screw instrumentation are minimized by better understanding of the orientation of the pedicles during surgery and intra-operative image guidance. In both the groups early surgery results in less post-operative respiratory complications and therefore, reduced hospital stays. Early surgery in DMD also avoids pelvic instrumentation since pelvic obliquity was usually less than $10^{\circ}$. The percentage curve correction was significantly higher (83\% vs. $62 \%$ ) in the PS group as compared to SLW group. PS system appeared to retain the correction achieved at final follow-up.

Although the radiographic parameters show quite significant change in the PS group as compared to SLW group, the functional outcome remains similar in both groups. This is owing to the fact that both the techniques provide adequate support to the spine for a better sitting balance and may prevent further deterioration of pulmonary function. We recommend that pedicle instrumentation has better inherent capability of maintaining the corrected curve due to its biomechanical superiority.

The strengths of our study include single centre study, matching cohort of patients, availability of pre and post operative data, involvement of an independent assessor and critical analysis of the data. There were few limitations. First limitation, it's comparing a historical surgical outcome study from two different decades. Second limitation, the SLW group had pelvic fixation while the PS group had no pelvic fixation. Third limitation, since curve flexibility index was a prerequisite to compare the percentage correction, immediate post-operative correction was subtracted from the flexibility index in this series for interpreting the results.

\section{Conclusions}

Spinal instrumentation with pedicle screws for scoliosis correction in patients with DMD results in a good correction of the spinal and pelvic deformity. Pedicle instrumentation gives a solid foundation, on which the rest of the spine may be held upright, maintain the balance and rotation. Due to the improvement of lumbar curve and pelvic obliquity by pedicle instrumentation, a good sitting balance is restored even without the pelvic or sacral fixation in patients with pelvic obliquity less than $10^{\circ}$.

\section{REFERENCES}

1. Cambridge W, Drennan JC. Scoliosis associated with Duchenne muscular dystrophy. J Pediatr Orthop 1987;7:436-40.

2. Galasko CS, Williamson JB, Delaney CM. Lung function in Duchenne muscular dystrophy. Eur Spine J 1995;4:263-7.

3. Hsu JD. The natural history of spine curvature progression in the nonambulatory Duchenne muscular dystrophy patient. Spine (Phila Pa 1976) 1983;8:771-5.

4. Bellen P, Hody JL, Clairbois J, Denis N, Soudon P. The surgical treatment of spinal deformities in Duchenne muscular dystrophy. J Orthop Surg 1993;7:48-57.

5. Bridwell KH, Baldus C, Iffrig TM, Lenke LG, Blanke K. Process measures and patient/parent evaluation of surgical management of spinal deformities in patients with progressive flaccid neuromuscular scoliosis (Duchenne's muscular dystrophy and spinal muscular atrophy). Spine (Phila Pa 1976) 1999;24:1300-9.

6. Harrington PR. Treatment of scoliosis: correction and internal fixation by spine instrumentation. J Bone Joint Surg Am 1962;44:591-610.

7. Heller KD, Wirtz DC, Siebert CH, Forst R. Spinal stabilization in Duchenne muscular dystrophy: principles of treatment and record of 31 operative treated cases. J Pediatr Orthop B 2001;10:18-24.

8. Luque ER. The anatomic basis and development of segmental spinal instrumentation. Spine (Phila Pa 1976) 1982;7:256-9.

9. Miller F, Moseley CF, Koreska J. Spinal fusion in Duchenne muscular dystrophy. Dev Med Child Neurol 1992;34:775-86.

10. Mubarak SJ, Morin WD, Leach J. Spinal fusion in Duchenne muscular dystrophy: fixation and fusion to the 
sacropelvis? J Pediatr Orthop 1993;13:752-7.

11. Rideau Y, Glorion B, Delaubier A, Tarle O, Bach J. The treatment of scoliosis in Duchenne muscular dystrophy. Muscle Nerve 1984;7:281-6.

12. Sussman MD. Advantage of early spinal stabilization and fusion in patients with Duchenne muscular dystrophy. J Pediatr Orthop 1984;4:532-7.

13. Swank SM, Brown JC, Perry RE. Spinal fusion in Duchenne' s muscular dystrophy. Spine 1982;7:484-91.

14. Yazici M, Asher MA, Hardacker JW. The safety and efficacy of Isola-Galveston instrumentation and arthrodesis in the treatment of neuromuscular spinal deformities. J Bone Joint Surg Am 2000;82:524-43.

15. Alman BA, Kim HK. Pelvic obliquity after fusion of the spine in Duchenne muscular dystrophy. J Bone Joint Surg Br 1999;81:821-4.

16. Gaine WJ, Lim J, Stephenson W, Galasko CS. Progression of scoliosis after spinal fusion in Duchenne's muscular dystrophy. J Bone Joint Surg Br 2004;86:550-5.

17. Sengupta DK, Mehdian SH, McConnell JR, Eisenstein SM, Webb JK. Pelvic or lumbar fixation for the surgical management of scoliosis in duchenne muscular dystrophy. Spine (Phila Pa 1976) 2002;27:2072-9.

18. Barr SJ, Schuette AM, Emans JB. Lumbar pedicle screws versus hooks: results in double major curves in adolescent idiopathic scoliosis. Spine (Phila Pa 1976) 1997;22:136979.

19. Liljenqvist U, Lepsien U, Hackenberg L, Niemeyer T, Halm H. Comparative analysis of pedicle screw and hook instrumentation in posterior correction and fusion of idiopathic thoracic scoliosis. Eur Spine J 2002;11:336-43.

20. Min K, Waelchli B, Hahn F. Primary thoracoplasty and pedicle screw instrumentation in thoracic idiopathic scoliosis. Eur Spine J 2005;14:777-82.

21. Hahn F, Hauser D, Espinosa N, Blumenthal S, Min K. Scoliosis correction with pedicle screws in Duchenne muscular dystrophy. Eur Spine J 2008;17:255-61.

22. Oda T, Shimizu N, Yonenobu K, Ono K, Nabeshima T, Kyoh S. Longitudinal study of spinal deformity in Duchenne muscular dystrophy. J Pediatr Orthop 1993; 13:478-88.

23. Steffensen B, Hyde S, Lyager S, Mattsson E. Validity of the EK scale: a functional assessment of non-ambulatory individuals with Duchenne muscular dystrophy or spinal muscular atrophy. Physiother Res Int 2001;6:119-34.

24. Cervellati S, Bettini N, Moscato M, Gusella A, Dema E, Maresi R. Surgical treatment of spinal deformities in Duchenne muscular dystrophy: a long term follow-up study. Eur Spine J 2004;13:441-8.

25. Allen BL Jr, Ferguson RL. The Galveston technique of pelvic fixation with L-rod instrumentation of the spine. Spine (Phila Pa 1976) 1984;9:388-94.

26. Arun R, Srinivas S, Mehdian SM. Scoliosis in Duchenne's muscular dystrophy: a changing trend in surgical management: a historical surgical outcome study comparing sublaminar, hybrid and pedicle screw instrumentation systems. Eur Spine J 2010;19:376-83.

27. Gayet LE, Duport G, Pries P. Flexible and semi-early vertebral instrumentation in surgical treatment of Duchenne muscular dystrophy scoliosis. Eur J Orthop Surg Traumatol 1999;9:223-31.

28. Hitchon PW, Brenton MD, Black AG, et al. In vitro biomechanical comparison of pedicle screws, sublaminar hooks, and sublaminar cables. J Neurosurg 2003;99(1 Suppl):1049.

29. Suk SI, Kim WJ. Biomechanics in posterior spinal instrumentation in biomechanics and biomaterials. In: Poitout DG, editor. Orthopaedics. Berlin: Springer; 2004. p. 462-91.

30. Kim YJ, Lenke LG, Kim J, et al. Comparative analysis of pedicle screw versus hybrid instrumentation in posterior spinal fusion of adolescent idiopathic scoliosis. Spine (Phila Pa 1976) 2006;31:291-8. 